\title{
Physical and Mechanical Properties Evaluation of Particle Board Produced from Saw Dust and Plastic Waste
}

\author{
Atoyebi Olumoyewa Dotun *, Adeolu Adesoji Adediran, Adisa Cephas Oluwatimilehin
}

The current work reports on the fabrication of composite matrix from saw dust (SD) and recycled polyethylene terephthalate (PET) at different weight ratio by flat-pressed method. Wood plastic composites (WPCs) were made with a thickness of $15 \mathrm{~mm}$ after mixing the saw dust and PET followed by a three phase press cycle. Physical properties (Density, Water Absorption (WA) and Thickness Swelling (TS)) and Mechanical properties (Modulus of Elasticity (MOE) and Modulus of Rupture (MOR)) were determined base on the mixing ratios according to the standard. WA and TS were measured after $2 \mathrm{~h}$ and $24 \mathrm{~h}$ of immersion in water. The results showed that as the density increased, the SD content decreased from $90 \%$ to $50 \%$ into the matrix. However, WA and TS decreases when the PET content increased in the matrix. Remarkably, the MOE and MOR attained a maximum point at $964.199 \mathrm{~N} / \mathrm{mm}^{2}$ and $9.03 \mathrm{~N} / \mathrm{mm}^{2}$ respectively in $50 \% \mathrm{SD}$ content. In comparism with standard, boards D and E can be classified as medium density boards while A, B and $\mathrm{C}$ are low density boards. The results indicated that the fabrication of WPCs from sawdust and PET would technically be feasible for indoor uses in building due to favorable physical properties exhibited. The mechanical properties response showed that it cannot be used for structural or load bearing application. 